\title{
HIV-1 Vif up-regulates the expression of Tat via AKT Signaling pathway: Role of deubiquitinase USP17 and NQ01
}

Sneh Lata ( $\sim$ snehlataviro@gmail.com )

National Institute of Immunology

Vikas Sood

Akhil Banerjea

National Institute of Immunology

\section{Article}

Keywords: HIV-1, Tat, AKT, Vif, phosphorylation, NQ01, DUB, Mdm2, USP17

Posted Date: October 1st, 2021

DOI: https://doi.org/10.21203/rs.3.rs-944391/v1

License: (a) (i) This work is licensed under a Creative Commons Attribution 4.0 International License. Read Full License 


\section{Abstract}

Human Immunodeficiency Virus-1 (HIV-1) has a small RNA genome and depends on host cellular machinery for most of its activities. Host cellular proteins modulate the expression and activity of viral proteins to combat the virus. HIV-1 proteins are known to regulate each other for the benefit of virus by exploiting these modulations. Here, we report that HIV-1 Vif increases the expression of Tat via AKT signaling pathway. We show that HIV-1 Vif activates AKT signaling pathway by inducing phosphorylation of AKT. Mdm2, downstream target of AKT signaling, increases the expression of Tat protein in ubiquitinindependent manner by up-regulating NQO1 as well as in ubiquitin-dependent manner by inducing the expression levels of USP17 which is a deubiquitinase (DUB) and stabilizes Tat protein. Thus, HIV-1 proteins exploit AKT signaling pathway to escape host restriction factors and promote viral replication.

\section{Introduction}

HIV-1 is a small retrovirus having two copies of single stranded RNA genome. It encodes for three structural proteins viz. Gag, Pol and Env and six accessory proteins viz. Tat, Rev, Vif, Vpr, Vpu and Nef ${ }^{1}$. These proteins interacts with many cellular proteins and hijacks cellular machinery for promoting viral replication by overcoming various host restriction factors ${ }^{2,3}$. HIV-1 Nef down-regulates CD4 (cluster of differentiation 4$)^{4}$ and MHC- 1 (major histocompatibility complex-1) ${ }^{5}$ to evade innate immune recognition and to prevent super-infection. HIV-1 Vif down-regulates APOBEC3G (apolipoprotein B mRNA-editing enzyme-catalytic polypeptide-like $3 G$ ) by proteasomal degradation ${ }^{6}$. Vpu down-regulates tetherin in beta$\operatorname{TrCP}$ (beta-transducin repeat containing protein) dependent manner ${ }^{7}$.

HIV-1 is known to induce the activation of PI3/AKT signaling pathway and this effect is mediated by Tat protein $^{8,9,10,11}$. Mdm2 is a downstream target of $A K T^{12,13}$. Previously, we have also shown that HIV-1 Tat protein stabilizes Mdm2 by inducing its phosphorylation in AKT dependent manner ${ }^{14}$. MDM2 is also known to enhance the Tat mediated LTR activity. MDM2 ubiquitinates Tat at lysine 71 position to potentiate its activity in a non-proteolytic way ${ }^{15}$. Thus, there is a positive feed-back loop between Tat, AKT and Mdm2. But MDM2 is an E3 ligase which has been shown to interact with HIV-1 Vif ${ }^{16}$, leading to its ubiquitination followed by its proteasomal degradation. Vif protein is required for efficient viral replication because it counteracts the host restriction factor APOBEC3G. APOBEC3G induces hypermutations in viral DNA by cytidine deaminase activity leading to degradation of viral DNA. If hypermutated viral DNA gets integrated in the cellular genome, it cannot code for functional viral proteins ${ }^{17}$. Vif recruits E3 ubiquitin ligase complex and decreases the levels of $A P O B E C 3 G$ protein via proteasomal degradation pathway ${ }^{6}$. Vif deficient viruses are severely compromised and unable to multiply in host cells ${ }^{18}$.

So, to investigate the mechanism how HIV-1 escapes this host anti-viral response of Mdm2 mediated degradation of Vif protein, the effect of HIV-1 Vif expression on AKT signaling pathway was examined. We found that Vif increased the phosphorylation of AKT on the one hand while it induced ubiquitinmediated proteasomal degradation of Mdm2 on the other hand. Since Tat is previously known to activate 
AKT signaling pathway $8,9,10,11$ and HIV-1 proteins are previously known to regulate each other by modulating the function of host proteins and support the viral replication viz. Vif degrades Vpr protein and reduces Vpr-mediated cell cell cycle arrest ${ }^{19}$, Rev induces the degradation of Tat in ubiquitinindependent manner via regulation of NQO1 $(\mathrm{NAD}(\mathrm{P}) \mathrm{H} \text { : Quinone Oxidoreductase } 1)^{20}$, so to investigate how HIV-1 is benefitted by activating AKT signaling pathway via its two proteins, Tat and Vif, despite the fact that Mdm2, which is activated by AKT, degrades Vif protein, we investigated the effect of Vif on the expression of Tat. We found that Vif increased the expression levels of Tat protein. Vif was also found to increase the LTR transcription mediated by Tat protein and viral replication. Vif also exhibited specific interaction with Tat and AKT forming a triplet Tat-AKT-Vif. Inhibition of AKT activity abrogated Vif mediated stabilization of Tat protein. Here, Mdm2 was found to increase the expression of Tat via NQ01 and a deubiquinase, USP17. Thus, AKT signaling pathway was playing an important role in the regulation of HIV-1 Tat by Vif via Mdm2 mediated stabilization of other host proteins NQ01 and USP17. This study can have significant implications towards better understanding the several mechanisms of HIV-1mediated exploitation of host machinery and viral pathogenesis.

\section{Results}

\section{Vif increases phosphorylation of AKT at Ser 473}

To investigate the effect of Vif on AKT signaling pathway, HEK-293T cells were transfected with HA AKT, HA Myr AKT and HA KD AKT along with Myc Vif. After 24 hrs of transfection, cells were lysed and analyzed by western blotting. Vif was increasing the expression of Myr AKT while there was no effect of Vif on the expression of wild type HA AKT as well as the kinase deficient KD AKT. Vif was also increasing the levels of phospho-AKT Ser 473 in Myr AKT and Myc Vif co-transfection experiment (Fig. 1a).

We also checked the endogenous levels of phospho-AKT Ser 473 in the presence of Vif. HEK-293T cells were transfected with increasing amounts of Myc Vif encoding plasmid. After $24 \mathrm{hrs}$ of transfection, cell lysates were analyzed by western blotting using anti-phospho-AKT Ser 473. It was observed that Vif was increasing the levels of phosphor-AKT Ser 473 in dose dependent manner while the expression of unmodified AKT was unaffected by Vif (Fig. 1b). This observation was also confirmed by using inhibitor of AKT activity or phosphorylation i.e. AKTi. HEK-293T cells were transfected with Myc Vif with or without AKTi treatment. After $24 \mathrm{hrs}$, cells were subjected to western analysis. AKTi was reducing the levels of phosho-AKT Ser 473 as expected. Vif was found to induce the phosphorylation of AKT at Ser 473 even in the presence of AKTi (Fig. 1c). These results indicate that HIV-1 Vif can increase the activity of AKT by inducing its phosphorylation at Ser 473.

\section{Vif induces ubiquitin-mediated proteasomal degradation of Mdm2}

Mdm2 is the downstream target of AKT. When PI3/AKT pathway is activated, AKT is phosphorylated and further it phosphorylates and stabilizes $M d m 2^{21}$. As, Vif was increasing the activity of AKT by inducing its phosphorylation, the effect of Vif on the levels of Mdm2 was also investigated. HEK-293T cells were 
co-transfected with HA Mdm2 and Myc Vif plasmids for $24 \mathrm{hrs}$. Western blotting analysis of these cells showed that Mdm2 is degraded in the presence of Vif (Fig. 1d). Vif was able to degrade Mdm2 at endogenous level also (Fig. 1e). The cycloheximide chase of Mdm2 alone and in the presence of Vif also showed that the stability of Mdm2 is reduced in the presence of Vif (Fig. 1f).

These results were confirmed using constitutively active form of AKT i.e. Myr AKT which stabilizes Mdm2 expression. HEK-293T cells were co-transfected with HA Mdm2, HA Myr AKT and Vif for $24 \mathrm{hrs}$ and cells were subjected to western analysis. As expected, Myr AKT was increasing the expression of Mdm2. Vif was found to reduce the expression level of Mdm2 even in the presence of Myr AKT which is itself increased by Vif (Fig. 1g). This result was also validated using inhibitor of AKT activity i.e. AKTi. Vif was increasing the levels of phospho-AKT Ser 473 even in the presence of AKTi as described above but the expression level of Mdm2 was found to be reduced in the presence of Vif (Fig. 1h).

To find out the mechanism of Vif-mediated degradation of Mdm2, HEK-293T cells were co-transfected with HA Mdm2 and Myc Vif in presence or absence of MG132 (proteasomal inhibitor). Vif-mediated degradation of Mdm2 was found to be reversed by MG132 (Fig. 1i). This indicates the post-translational regulation of $\mathrm{Mdm} 2$ by Vif. The ubiquitination levels of $\mathrm{Mdm} 2$ in presence of Vif were also checked. HEK293T cells were co-transfected with HA Mdm2, Myc Vif and His Ub plasmids. Cells were also treated with MG132 to accumulate ubiquitinated species. Cells were lysed and cell lysate was incubated with Ni-NTA beads which bind His tagged ubiquitinated proteins. The ubiquitination of Mdm2 was found to be increased in the presence of Vif (Fig. 1j). These results indicate that Vif induces ubiquitin-mediated proteasomal degradation of Mdm2.

\section{Vif increases expression of Tat and exhibits direct interaction}

The expression of Tat in the presence of Vif was investigated by co-transfecting HEK-293T cells with HA Tat and Myc Vif for 24hrs. Cell lysates were analyzed by western blotting to check the expression of Tat and Vif. Vif was found to stabilize the expression of Tat (Fig. 2a). Cycloheximide chase of Tat either alone or with Vif also showed that the stability of Tat is more in the presence of Vif (Fig.2b). As, Mdm2 is known to increase the LTR transcriptional activity of Tat ${ }^{15}$ and we are reporting the ubiquitin mediated proteasomal degradation of Mdm2 by Vif, the effect of Vif on LTR transcription mediated by Tat was also investigated by co-transfecting HEK-293T cells with B LTR luciferase reporter plasmid (pBlue3'LTR-luc) and Tat either alone or in combination with Vif encoding plasmid for $24 \mathrm{hrs}$. As expected, LTR activation was observed in the presence of Tat. Vif alone also showed little activation of LTR. Surprisingly, In the presence of both Vif and Tat proteins, LTR activation was observed to be much higher than that of Tat alone (Fig. 2c), indicating Vif mediated stabilization of Tat. Further, to find out the importance of these observations in context of HIV-1 virus, we co-transfected Vif deficient HIV-1 proviral DNA (pNL4-3 $\Delta$ Vif) into Tzm-BI cells either alone or along with Myc Vif for 24 hours. It was observed that the activation of

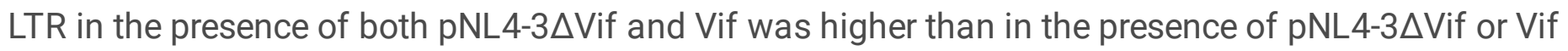
alone (Fig. 2d). 
The interaction between Tat and Vif was also investigated. In vitro synthesized HIV-1 Vif protein and GST Tat fusion protein were used to study their interaction. For in vitro binding studies, GST Tat and GST alone (control) were allowed to bind to GST beads. Thereafter, in vitro translated Vif protein was added to the complex and incubated for 2 hours at $4^{\circ} \mathrm{C}$. Vif protein was visualized on a nitro-cellulose membrane by western blot analysis using specific antiserum (obtained from NIH, MD, USA). GST Tat was able to pull down Vif specifically while GST alone failed to do so (Fig. 2e). This led to the conclusion that Vif specifically interacts with HIV-1 Tat protein. To further check the interaction between the three proteins viz. Tat, Vif and AKT, HEK-293T cells were transfected with HA Tat, Myc Vif and HA AKT. Cell lysates were subjected to immuno-precipitation with anti-Myc antibody tagged agarose beads and bound proteins were analyzed by western blotting using anti-HA antibody. Both proteins Tat and AKT were found to equally interact with Vif (Fig. 2f). These results indicate that Vif interacts with both Tat and AKT independently and can form triplet.

\section{Role of AKT siganaling pathway in HIV-1 Vif mediated stabilization of Tat}

As, Tat is known to play important role in activation of AKT signaling pathway and we are also reporting the up-regulation of phosphorylated AKT by Vif, so the role of AKT signaling pathway in Vif mediated stabilization of Tat was investigated using chemical inhibitor of AKT (AKTi). HEK-293T cells were transfected with HA Tat either alone or with Myc Vif and cells were treated with AKTi. Vif was observed to increase the expression of Tat protein as before but inhibition of AKT activity by AKTi was able to decrease the expression of Tat even in the presence of Vif (Fig. 3a). The effect of AKTi treatment on HIV-1 replication was also investigated in U1 cells (monocytes latently infected with HIV-1). HIV-1 replication was induced in $\mathrm{U} 1$ cells using PMA and cells were treated with AKTi. The inhibition of AKT activity by AKTi was found to reduce the HIV-1 replication (Fig. 3b). When Myr AKT, wild type AKT and KD AKT were co-expressed with HA Tat in HEK-293T cells, it was observed that the expression of Tat is downregulated in presence of both wild type AKT (Fig. 3c) and KD AKT (Fig. 3e) while there was no significant effect of Myr AKT on the Tat levels (Fig. 3d). This indicated the involvement of other host factor in stabilizing Tat which becomes functional upon activation of AKT signalling pathway and compensates for its downregulation by AKT.

Since Mdm2 is the immediate downstream target of AKT signalling pathway and also known to mediate the K63 ubiquitination of Tat by direct interaction resulting in the enhancement of LTR transcriptional activity of Tat and viral replication ${ }^{15}$, the expression of Tat in presence of Mdm2 was examined. HEK293T cells were co-transfected with HA Tat and HA Mdm2 for 24hrs. The expression of Tat protein was found to be enhanced in the presence of Mdm2 (Fig. 3f). Cycloheximide chase of Tat in the presence or absence of Mdm2 also showed that stability of Tat was increased in the presence of Mdm2 (Fig. $3 \mathrm{~g}$ ). These results indicated that Vif stabilizes the expression of Tat via Mdm2.

\section{Mdm2 increases the expression of NQO1 and vice versa}


As, we have previously reported that NQ01 stabilizes the expression of HIV-1 Tat protein ${ }^{20}$, so the expression of NQO1 in the presence of Mdm2 was also examined to investigate the mechanism of Mdm2 mediated stabilization of Tat. HEK-293T cells were co-transfected with Flag NQO1 and HA Mdm2 for 24hrs. The expression of NQ01 was found to be increased in the presence of Mdm2 (Fig. 4a). Mdm2 was found to increase the expression of NQ01 at endogenous level also (Fig. 4b). Cycloheximide chase of NQO1 in presence or absence of Mdm2 also showed that stability of NQO1 was enhanced in the presence of Mdm2 (Fig. 4c). The ubiquitination of NQO1 in presence of Mdm2 was also investigated. HEK-293T cells were co-transfected with Flag NQ01, HA Mdm2, and His Ub plasmids. Cells were also treated with MG132 to accumulate ubiquitinated species. Cells were lysed and cell lysate was incubated with Ni-NTA beads which bind His tagged ubiquitinated proteins. Ubiquitination levels of NQ01 were found to be downregualted in the presence of Mdm2 (Fig. 4d). To check the interaction between Mdm2 and NQ01, HEK-293T cells were transfected with Flag NQ01 and HA Mdm2 for 24 hrs and cell lysates were subjected to immunoprecipitation using anti-NQ01 antibody bound agarose beads. The western blot analysis of pulled down proteins using anti-HA antibody indicated the interaction between NQO1 and Mdm2 (Fig. 4e).

As NQ01 is known to stabilize the proteins by inhibiting their degradation by $20 \mathrm{~S}$ proteasome ${ }^{22}$, so the reverse regulation of Mdm2 by NQ01 was also checked. HEK-293T cells were co-transfected with HA Mdm2 and Flag NQO1 for 24hrs. The expression of Mdm2 was found to be increased in the presence of NQ01 (Fig. 4f). NQ01 was also found to stabilize Mdm2 protein at endogenous level (Fig. 4g). This observation was further confirmed by using diminutol (chemical inhibitor of NQ01 activity). HEK-293T cells were treated with different doses of diminutol for $6 \mathrm{hrs}$. The expression of Mdm2 was found to be down-regulated in the presence of diminutol (Fig. 4h). Cycloheximide chase of Mdm2 in presence of diminutol also confirmed that stability of $\mathrm{Mdm} 2$ is reduced in the presence of diminutol (Fig. 4i). These results indicated that NQ01 stabilizes the expression of Mdm2 by inhibiting its $20 \mathrm{~S}$ proteasomal degradation.

The expression of NQO1 in the presence of AKTi was also investigated. Inhibition of AKT activity was found to reduce the expression level of NQO1 in HEK-293T cells. This result was further validated in ThP1 cells (natural host of HIV-1). AKTi treatment was found to reduce the levels of NQO1 in ThP-1 cells also (Fig. 4j). These results indicated that activation of PI3/AKT signalling pathway up-regulates the expression of HIV-1 Tat protein via Mdm2 mediated stabilization of NQ01.

\section{Regulation of HIV-1 Tat expression via USP17 in ubiquitin-dependent manner}

To confirm the ubiquitin-independent up-regulation of Tat expression by Mdm2 via NQ01, the ubiquitination assay was performed. HEK-293T cells were transfected with Flag Tat, HA Mdm2 and His $\mathrm{Ub}$ plasmids for $24 \mathrm{hrs}$. Cells were treated with MG132 for $8 \mathrm{hrs}$ followed by immunoprecipitation using NiNTA resin. Western blot analysis was done to detect ubiquitinated species of Tat using anti-Flag antibody. Surprisingly, ubiquitination of Tat was found to be reduced in the presence of Mdm2 (Fig. 5a). This indicated the involvement of ubiquitination in Mdm2 mediated stabilization of Tat. 
As, we have previously reported the stabilization of HIV-1 Tat protein in ubiquitination dependent manner by USP7 which is a deubiquitinase ${ }^{23}$, so we explored the possibility of the involvement of a deubiquitinase in Mdm2 mediated stabilization of Tat. We found a deubiquitinase, USP17 which was stabilizing the expression of Tat when co-expressed in HEK-293T cells (Fig. 5b). Cycloheximide chase of Tat in presence of USP17 also indicated that stability of Tat is increased in the presence of USP17 (Fig. 5c). Ubiquitination of Tat was also found to be increased by USP17 (Fig. 5d). To check the interaction between Tat and USP17, HEK-293T cells were transfected with HA Tat and co-immunprecipitaion was performed using anti-USP17 antibody bound agarose beads. This showed that USP17 interacts with HIV1 Tat protein specifically (Fig. 5e). The regulation of USP17 expression by HIV-1 was also investigated in U1 cells. U1 cells were treated with PMA to activate latent virus for different time intervals followed by western blotting. The expression of USP17 was found to be induced by HIV-1 replication (Fig. 5f). These results indicated that USP17 stabilizes the expression of Tat in ubiquitin-dependent manner and is induced by HIV-1 infection also.

\section{Mdm2 increases the expression of USP17 via direct interaction}

The effect of Mdm2 on the expression of USP17 was investigated by co-expressing them in HEK-293T cells. The levels of USP17 were found to be increased in the presence of Mdm2 (Fig. 6a). The dosedependent effect of Mdm2 on the levels of USP17 was also observed (Fig. 6b). Mdm2 was stabilizing the expression of USP17 at endogenous level also (Fig. 6c). The ubiquitination of USP17 in presence of Mdm2 was also investigated. HEK-293T cells were co-transfected with His USP17, HA Mdm2, and His Ub plasmids. Cells were also treated with MG132 to accumulate ubiquitinated species. Cells were lysed and cell lysate was incubated with Ni-NTA beads which bind His tagged ubiquitinated proteins followed by western blotting using anti-USP17 antibody. Ubiquitination of USP17 was found to be reduced by Mdm2 indicating the ubiquitin-dependent regulation of USP17 expression by Mdm2 (Fig. 6d). To check the interaction between Mdm2 and USP17, cell lysates of HEK-293T cells were subjected to coimmunoprecipitation using anti-Mdm2 antibody bound agarose resin followed by western blotting. Mdm2 was found to interact with USP17 specifically (Fig. 6e). To further confirm Mdm2-mediated stabilization of USP17, Mdm2 was downregulated using specific siRNA in HEK-293T cells. The expression of USP17 was found to be reduced in the presence of Mdm2 siRNA (Fig. 6f). The expression of USP17 in the presence of AKTi was also investigated. Inhibition of AKT activity was found to reduce the expression level of USP17 in HEK-293T cells. This result was further validated in ThP-1 cells. AKTi treatment was found to reduce the levels of USP17 in ThP-1 cells also (Fig. 6g). These results indicated that Mdm2 stabilizes Tat protein via inducing the expression of USP17.

\section{Discussion}

There are number of restriction factors which protect the host cells from the pathogen. HIV-1 has evolved various mechanisms to counter these host cell factors. The well-known anti-HIV-1 restriction factors are TRIM5 (tripartite motif 5) ${ }^{24}$, APOBEC3G, SAMHD1 (SAM and HD domain containing deoxy-ribonucleoside triphosphate triphosphohydrolase 1$)^{25}$, Tetherin or BST-2 (bone marrow stromal antigen-2) ${ }^{26}$. HIV-1 has 
developed various strategies to evade these restriction factors except TRIM5 as none of the HIV-1 proteins is known to target this. The restriction factors SAMHD1, Tetherin and APOBEC3G are targeted by $\mathrm{Vpx} / \mathrm{Vpr}, \mathrm{Vpu}$ (or Env) and Vif respectively ${ }^{27}$. HIV-1 Vif-mediated APOBEC3G degradation ${ }^{6}$ is essential for viral replication. However, there are very few reports about the regulation of Vif protein. Core Binding Factor $\beta$ (CBF $\beta$ ) has been shown to stabilize Vif with subsequent effects on APOBEC3G levels ${ }^{28}$. MDM2 is reported to promote Vif degradation to elevate APOBEC3G levels. As, Mdm2 is a downstream target of $\mathrm{AKT}^{12,13}$ which is reported to be induced by HIV-1 Tat ${ }^{8,9,10,11}$, so to find out how HIV-1 cope up with this host anti-viral response, we investigated the effect Vif on AKT signaling pathway. Vif was observed to increase the levels of phospho-AKT Ser 473 while it was found to induce ubiquitin-mediated proteasomal degradation of Mdm2. As, HIV-1 Tat is already known to activate AKT signaling pathway 8, 9, 10,11, so the effect of Vif on the expression of Tat was investigated to find out the role of AKT signaling pathway in HIV-1 pathogenesis. Vif was found to increase the levels of Tat protein and Tat-mediated LTR transactivation was also found to be enhanced in the presence of Vif. The inhibition of AKT activity was interfering with Vif mediated increase in Tat expression but there was no effect of Myr-AKT on the expression of Tat while wild type AKT and KD-AKT were down-regulating the Tat levels. So, we hypothesized that there is another host factor which stabilizes Tat upon activation of AKT signaling pathway. Mdm2, a downstream target of AKT was found to induce an increase in the expression of Tat. When mechanism of Mdm2 mediated stabilization of Tat was investigated, it was observed that Mdm2 can up-regulate the expression of HIV-1 Tat protein via NQ01 which stabilizes the expression of Tat in ubiquitin independent manner as well as USP17 which stabilizes Tat by deubiquitinating it. These results indicated the important role of AKT signaling pathway in regulation of HIV-1 protein expression and viral replication.

The exploitation and modulation of host cellular signaling pathways by HIV-1 proteins is a very complex phenomenon. HIV-1 Tat is an early protein of the virus which also activates AKT signaling pathway 8, 9, 10, 11. Tat also phosphorylates Mdm2 via AKT resulting in its stabilization as previously shown by us ${ }^{14}$ and Mdm2 further increases the expression of Tat as shown above and also increases the LTR transcriptional activity of Tat by inducing its non-proteolytic $\mathrm{K} 63$ ubiquitination ${ }^{15}$ thus creating a positive feedback loop between Tat, AKT and Mdm2. On the one hand, HIV-1 Vif also increases phosphorylation of AKT which increases Tat expression and viral replication via Mdm2. On the other hand, Vif induces proteasomal degradation of Mdm2 and reduces the levels of Mdm2 which is activated by AKT itself $\mathrm{f}^{12,13}$ and degrades $\mathrm{Vif}^{6}$ creating a negative feedback loop between Vif and Mdm2 (Fig. 7). Thus HIV-1 exploits AKT signaling pathway through its activation by Tat and Vif which results in the enhancement of Tat expression levels while keeping a check on the levels of Mdm2 via Vif to overcome its degradation by Mdm2. It helps the virus to evade the host restriction machinery more effectively and survive in the host cell.

\section{Materials And Methods}

\section{Cell culture and transfection}


HEK-293T (Human Embryonic Kidney 293T)and Tzm-BI cells were maintained in Dulbecco's Modified Eagle's Medium (DMEM; Himedia Laboratories) supplemented with $10 \%$ fetal bovine serum (Gibco, Invitrogen), 100 units of penicillin, $0.1 \mathrm{mg}$ streptomycin and $0.25 \mu \mathrm{g}$ amphotericin $\mathrm{B}$ per $\mathrm{ml}$ at $37^{\circ} \mathrm{C}$ in presence of $5 \% \mathrm{CO}_{2}$ in a humidified incubator. Thp- 1 and $\mathrm{U} 1$ cells were maintained in RPMI-1640 supplemented with $10 \%$ fetal bovine serum (Gibco, Invitrogen), 100 units of penicillin, $0.1 \mathrm{mg}$ streptomycin and $0.25 \mu \mathrm{g}$ amphotericin $\mathrm{B}$ per $\mathrm{ml}$ at $37^{\circ} \mathrm{C}$ in presence of $5 \% \mathrm{CO}_{2}$ in a humidified incubator. Transfections were performed using Lipofectamine 2000 (Invitrogen, USA) and Polyethyleneimine (PEI), Linear (MW 25,000, Polysciences Inc., USA) reagents using the manufacturer's protocol.

\section{Plasmid constructs and chemicals}

Plasmid Myc Vif was made by cloning pNL4-3 derived gene vif in pCMV-Myc plasmid from Clontech, USA as described earlier (Arora et al., 2014). HA APOBEC3G was cloned using cDNA from TZMbl cells in pCMV-HA vector from Clontech, USA. pBlue3'LTR-luc was obtained from NIH AIDS Reference and Reagent Program of NIH, MD, USA. GST Tat was generated by cloning pNL4-3 derived tat gene in pGEX-4T1 vector from Addgene. HA Tat, Flag Tat and Flag NQ01 were purchased from Addgene. HA Mdm2 was purchased from Sino Biologicals, USA. HA AKT, HA KD AKT (K179A), HA Myr AKT, GST AKT were a kind gift from Hui Kuan Lin, MD Anderson cancer centre, Texas. pNL4-3.Luc.R.E. was gifted by Nathaniel Landau (Rockefeller University, USA). Renilla luciferase plasmid was a kind gift from Vivek Natrajan, IGIB, Delhi, India. His Ub plasmid was gifted by Dimitris Xirodimas, University of Dundee. Chemicals used were AKTi (Sigma), PMA (Sigma), Diminutol (Sigma), IPTG (Sigma), Cycloheximide (Sigma) and MG132 (Sigma).

\section{Western blot analysis}

HEK-293T cells were transfected with gene of interest for $36 \mathrm{hrs}$. The the cells were harvested and lysed in RIPA lysis buffer (1\% NP-40, 20mM TrisCl, pH 7.5, $150 \mathrm{mM} \mathrm{NaCl}, 1 \mathrm{mM} \mathrm{Na}{ }_{2}$ EDTA, $1 \mathrm{mM}$ EGTA, $1 \%$ Sodium deoxycholate, $1 \mathrm{mM} \mathrm{Na}_{3} \mathrm{VO}_{4}$ ). Protein estimation was carried out using BCA Protein Assay Kit (Pierce, Thermo Scientific, USA). An equal amount of protein was loaded on SDS-PAGE and was transferred to nitrocellulose membrane. The membranes were blocked with $5 \%$ non-fat dry milk (Himedia Laboratories, India). The primary antibodies used were anti-AKT, anti-Mdm2, anti-AKT substrate, antiGAPDH, anti-phospho-AKT (S473) (Cell Signaling Technology), anti-myc, anti-HA (Clontech), anti-GST (Santa Cruz Biotechnology) and anti-Vif (NIH, MD, USA). The secondary antibodies used were antirabbit/mouse-HRP conjugated (Jackson Immuno Research). Blots were developed using ECL (Enhanced Chemiluminescence) reagent.

\section{Cycloheximide chase assay}

To study the degradation kinetics of proteins, cycloheximide chase assay was performed. HEK-293T cells were transfected with gene of interest for $24 \mathrm{hrs}$ and treated with CHX $(100 \mu \mathrm{g} / \mathrm{ml}$; Sigma). Cell lysates were prepared at indicated time points and subjected to $10 \%$ SDS-PAGE followed by western analysis as described above. 


\section{In vitro ubiquitination assay}

In vivo ubiquitination assay was performed to detect ubiquitylated proteins in transfected mammalian cells. HEK-293T cells were co-transfected with plasmid encoding desired gene and His-Ub (6X Histidine-

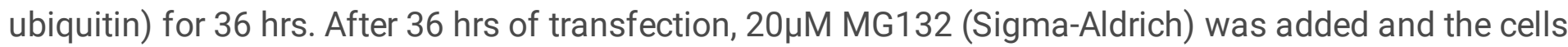
were further incubated for $8 \mathrm{hrs}$. The cells were lysed in Buffer A (6M guanidinium- $\mathrm{HCl}, 0.1 \mathrm{M}$ $\mathrm{Na}_{2} \mathrm{HPO}_{4} / \mathrm{NaH}_{2} \mathrm{PO}_{4}, 10 \mathrm{mM}$ imidazole; $\mathrm{pH}$ 8.0), sonicated, and centrifuged. Ni-NTA beads were added to the supernatant and the mixture was incubated at room temperature for 6 hrs while rotating. Subsequently, the beads were washed with buffer A and buffer TI (25mM Tris, pH 6.8, 20mM imidazole). The ubiquitinated proteins were eluted in buffer containing $200 \mathrm{mM}$ imidazole, $5 \%$ SDS, $0.15 \mathrm{M}$ Tris, $\mathrm{pH}$ $6.7,30 \%$ glycerol, and $0.72 \mathrm{M} \beta$-mercaptoethanol. The eluates were resolved by SDS-PAGE followed by western analysis.

\section{GST-fusion protein expression and purification}

pGEX-4T1 vector containing the desired gene was transformed in BL21 strain of $E$. Coli for expression and subsequent purification. The bacterial culture was induced with $0.5 \mathrm{mM}$ IPTG for 16 hours at $16^{\circ} \mathrm{C}$. The cells were lysed by adding lysozyme $(1 \mathrm{mg} / \mathrm{ml})$ at $4^{\circ} \mathrm{C}$ with gentle shaking. DTT was added to the bacterial lysate after lysozyme treatment $(100 \mu$ l of $1 \mathrm{M} \mathrm{DTT})$. This was followed by sonication and extraction of proteins with Triton X-100. The solution was centrifuged at 12,000 rpm for 15 minutes at $4^{\circ} \mathrm{C}$. The supernatant was then used for binding to glutathione beads at $4^{\circ} \mathrm{C}$ for 3 hours. The beads were centrifuged at $2500 \mathrm{rpm}$ for 2 minutes at $4^{\circ} \mathrm{C}$. The beads were washed till the supernatant stops giving color with Bradford reagent. A control with GST only, bound to glutathione beads was expressed and purified in similar way.

\section{GST-Pull down assay}

GST alone and GST tagged proteins were expressed and purified as described above. HEK-293T cells were transfected with gene of interest for 36 hours. The cells were lysed in RIPA buffer. 10ug of GST tagged protein was incubated with the cell lysate for 4 hours at $4^{\circ} \mathrm{C}$. After incubation, the supernatant was discarded and the beads were washed 5-6 times with chilled 1 PBS. The beads were boiled in laemmli buffer and subjected to SDS-PAGE followed by immunoblotting with anti-Myc and anti-GST antibodies.

\section{Immunoprecipitation}

The protein-protein interaction was studied by immunoprecipitation. The genes of interest were cotransfected in HEK-293T cells. Twenty hours post transfection, cell lysates were prepared in CelLytic M, cell lysis reagent (Sigma) and were incubated with anti-Myc agarose beads (Sigma) at $4^{\circ} \mathrm{C}$ overnight. The beads were washed with IP buffer (Sigma). The purified protein complex bound to anti-Myc agarose beads was resolved by SDS-PAGE and subjected to western blot analysis. 


\section{Dual Luciferase Reporter Assay}

Luciferase reporter assay was performed using the Dual-Luciferase Reporter assay kit (Promega, USA). HEK-293T cells were co-transfected with luciferase reporter plasmid and the plasmids encoding genes of interest. Renilla luciferase was used as control to normalize the transfection efficiency. Empty pcDNA3.1 vector was used to equalize the amount of DNA transfected in each well. After $24 \mathrm{hrs}$ of transfection, cell were harvested and lysed in lysis buffer (Promega, USA). Luciferase activity was measured by luminometer (Tecan, Switzerland) using two substrates (Promega, USA): one for firefly luciferase and another for renilla luciferase (mixed with stop and glo buffer). The readings of firefly luciferase activity were normalized with that of renilla luciferase activity to get the true luciferase reporter activity.

\section{Statistical analysis}

Results obtained were represented as mean \pm standard error of the mean (s.e.m). p values were calculated by a two-tailed t-test. Only values with $p<0.05$ were considered significant.

\section{Declarations}

\section{Acknowledgements:}

Several reagents were obtained from AIDS Reference \& Reagent Program of NIH, MD, USA. We thank Hui Kuan Lin (MD Anderson cancer centre, Texas) for HA AKT, HA KD AKT (K179A) and HA Myr AKT, GST AKT, Nathaniel Landau (Rockefeller University, USA) for pNL4-3.Luc.R.E., Vivek Natrajan (IGIB, Delhi, India) for renilla luciferase plasmid and Dimitris Xirodimas (University of Dundee) for His Ub plasmid. This work was supported by Department of Biotechnology and Department of Science and Technology of Government of India.

\section{References}

1. Frankel, A.D. and Young, J.A.T. (1998) HIV-1: fifteen proteins and an RNA. Annu. Rev. Biochem. 67, 125.

2. Emerman, M. \& Malim, M. H. HIV-1 regulatory/accessory genes: keys to unraveling viral and host cell biology. Science 280, 1880-1884 (1998).

3. Malim, M.H. and Emerman, M. (2008) HIV-1 accessory proteins-ensuring viral survival in a hostile environment. Cell Host Microbe 3, 388-398

4. Chaudhuri R, Lindwasser OW, Smith WJ, Hurley JH, Bonifacino JS. Downregulation of CD4 by human immunodeficiency virus type $1 \mathrm{Nef}$ is dependent on clathrin and involves direct interaction of Nef with the AP2 clathrin adaptor. J Virol. 2007 Apr;81(8):3877-90.

5. Blagoveshchenskaya AD, Thomas L, Feliciangeli SF, Hung CH, Thomas G. HIV-1 Nef downregulates MHC-I by a PACS-1- and PI3K-regulated ARF6 endocytic pathway. Cell. 2002 Dec 13;111(6):853-66. 
6. Yu, X., Yu, Y., Liu, B., Luo, K., Kong, W., Mao, P., and Yu, X. F. (2003). Induction of APOBEC3G ubiquitination and degradation by an HIV-1 Vif-Cul5-SCF complex. Science 302, 1056-1060.

7. Douglas JL, Viswanathan K, McCarroll MN, Gustin JK, Früh K, Moses AV. Vpu directs the degradation of the human immunodeficiency virus restriction factor BST-2/Tetherin via a \{beta\}TrCP-dependent mechanism. J Virol. 2009 Aug;83(16):7931-47.

8. Chugh, P., Fan, S., Planelles, V., Maggirwar, S.B., Dewhurst, S. and Kim, B. Infection of human immunodeficiency virus and intracellular viral Tat protein exert a pro-survival effect in a human microglial cell line. J. Mol. Biol. 366, 67-81 (2001).

9. Chugh, P., Bradel-Tretheway, B., Monteiro-Filho, C.M., Planelles, V., Maggirwar, S.B., Dewhurst, S., et al. Akt inhibitors as an HIV-1 infected macrophage-specific anti-viral therapy. Retrovirology 5, 11 (2008).

10. Deregibus, M.C., Cantaluppi, V., Doublier, S., Brizzi, M.F., Deambrosis, I., Albini, A., et al. HIV-1-Tat protein activates phosphatidylinositol 3-kinase/ AKT-dependent survival pathways in Kaposi's sarcoma cells. J. Biol. Chem. 277, 25195-25202 (2002).

11. Borgatti, P., Zauli, G., Colamussi, M.L., Gibellini, D., Previati, M., Cantley, L.L., et al. Extracellular HIV-1 Tat protein activates PI3K and Akt kinase in CD4+ T lymphoblstoid jurkat clls. Eur. J. Immunol. 27, 2805-2811 (1997).

12. Cooray, S. The pivotal role of phosphatidylinositol 3-kinase-Akt signal transduction in virus survival. J. Gen. Virol. 85, 1065-1076 (2004).

13. Manning, B.D. and Cantley, L.C. AKT/PKB signalling: navigating downstream. Cell 129, 1261-1274 (2007).

14. Raja R, Ronsard L, Lata S, Trivedi S, Banerjea AC. HIV-1 Tat potently stabilises Mdm2 and enhances viral replication. Biochem J. 2017 Jul 11;474(14):2449-2464.

15. Bres, V., Kiernan, R. E., Linares, L. K., Chable-Bessia, C., Plechakova, O., Treand, C., Emiliani, S., Peloponese, J. M., Jeang, K. T., Coux, O., et al. (2003). A non-proteolytic role for ubiquitin in Tatmediated transactivation of the HIV-1 promoter. Nat Cell Biol 5, 754-761.

16. Izumi, T., Takaori-Kondo, A., Shirakawa, K., Higashitsuji, H., Itoh, K., Io, K., Matsui, M., Iwai, K., Kondoh, H., Sato, T., et al. (2009). MDM2 is a novel E3 ligase for HIV-1 Vif. Retrovirology 6, 1.

17. Zhang, H., Yang, B., Pomerantz, R. J., Zhang, C., Arunachalam, S. C., and Gao, L. (2003). The cytidine deaminase CEM15 induces hypermutation in newly synthesized HIV-1 DNA. Nature 424, 94-98.

18. Goila-Gaur, R., and Strebel, K. (2008). HIV-1 Vif, APOBEC, and intrinsic immunity. Retrovirology 5, 51.

19. Wang, J. et al. The HIV-1 Vif protein mediates degradation of Vpr and reduces Vpr-induced cell cycle arrest. DNA Cell Biol. 27, 267-277 (2008).

20. Lata S, Ali A, Sood V, Raja R, Banerjea AC. (2015) HIV-1 Rev downregulates Tat expression and viral replication via modulation of $\mathrm{NAD}(\mathrm{P}) \mathrm{H}$ :quinine oxidoreductase 1 (NQ01). Nat. Commun. 6:7244.

21. Feng, J., Tamaskovic, R., Yang, Z., Brazil, D.P., Merlo, A., Hess, D. et al. (2004) Stabilization of Mdm2 via decreased ubiquitination is mediated by protein kinase B/Akt-dependent phosphorylation. J. Biol. Chem. 279, 35510-35517. 
22. Moscovitz O, Tsvetkov P, Hazan N, Michaelevski I, Keisar H, Ben-Nissan G, Shaul Y, Sharon M. A mutually inhibitory feedback loop between the $20 \mathrm{~S}$ proteasome and its regulator, NQ01. Mol Cell. 2012; 47(1):76-86.

23. Ali A, Raja R, Farooqui SR, Ahmad S, Banerjea AC. USP7 deubiquitinase controls HIV-1 production by stabilizing Tat protein. Biochem J. 2017 May 4;474(10):1653-1668.

24. Ganser-Pornillos BK, Pornillos O. Restriction of HIV-1 and other retroviruses by TRIM5. Nat Rev Microbiol. 2019 Sep;17(9):546-556.

25. St Gelais C, de Silva S, Amie SM, Coleman CM, Hoy H, Hollenbaugh JA, Kim B, Wu L. SAMHD1 restricts HIV-1 infection in dendritic cells (DCs) by dNTP depletion, but its expression in DCs and primary CD4+ T-lymphocytes cannot be upregulated by interferons. Retrovirology. 2012 Dec 11;9:105.

26. Le Tortorec A, Willey S, Neil SJ (May 2011). "Antiviral inhibition of enveloped virus release by tetherin/BST-2: action and counteraction". Viruses. 3 (5): 520-40.

27. Malim, M. H., and Bieniasz, P. D. 2012 HIV Restriction Factors and Mechanisms of Evasion. Cold Spring Harb Perspect Med 2, a006940.

28. Jäger, S., Kim, D. Y., Hultquist, J. F., Shindo, K., LaRue, R. S., Kwon, E., et al. (2011). Vif hijacks CBF-b to degrade APOBEC3G and promote HIV-1 infection. Nature 481, 371-375.

\section{Figures}


(a) Myc Vif

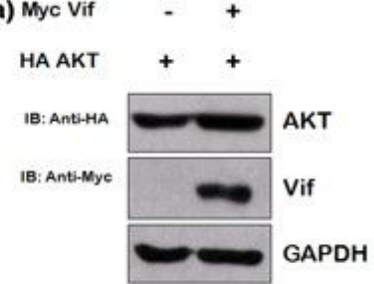

(c)

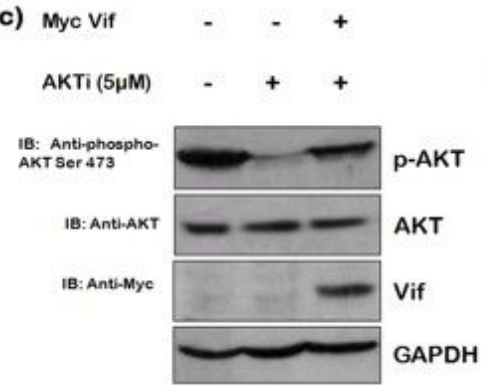

(g)
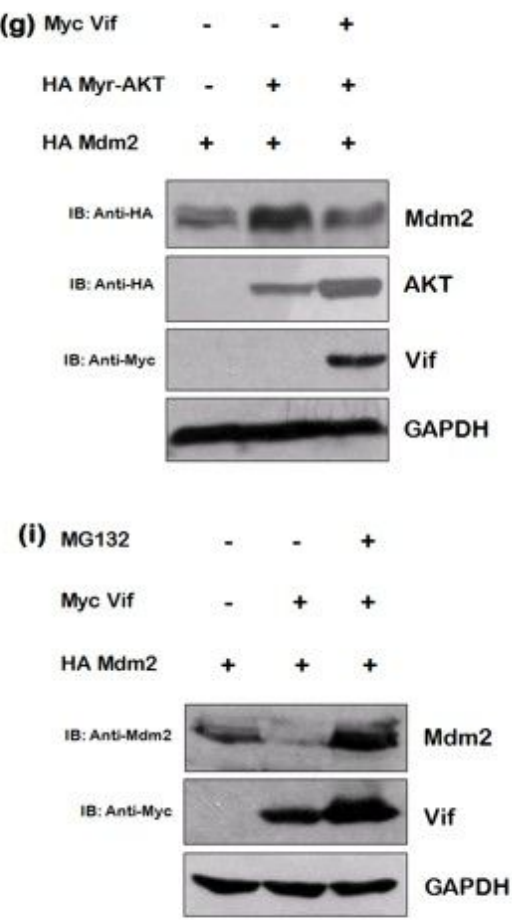

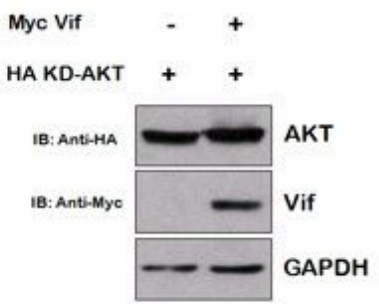

GAPDH (b)

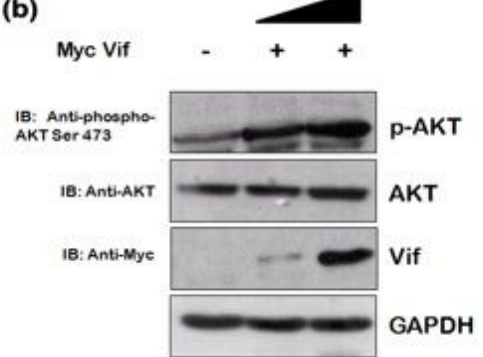

(d) Myc Vif

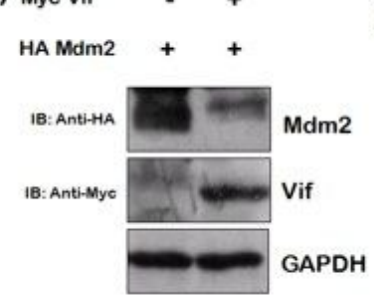

(e) Myc Vif
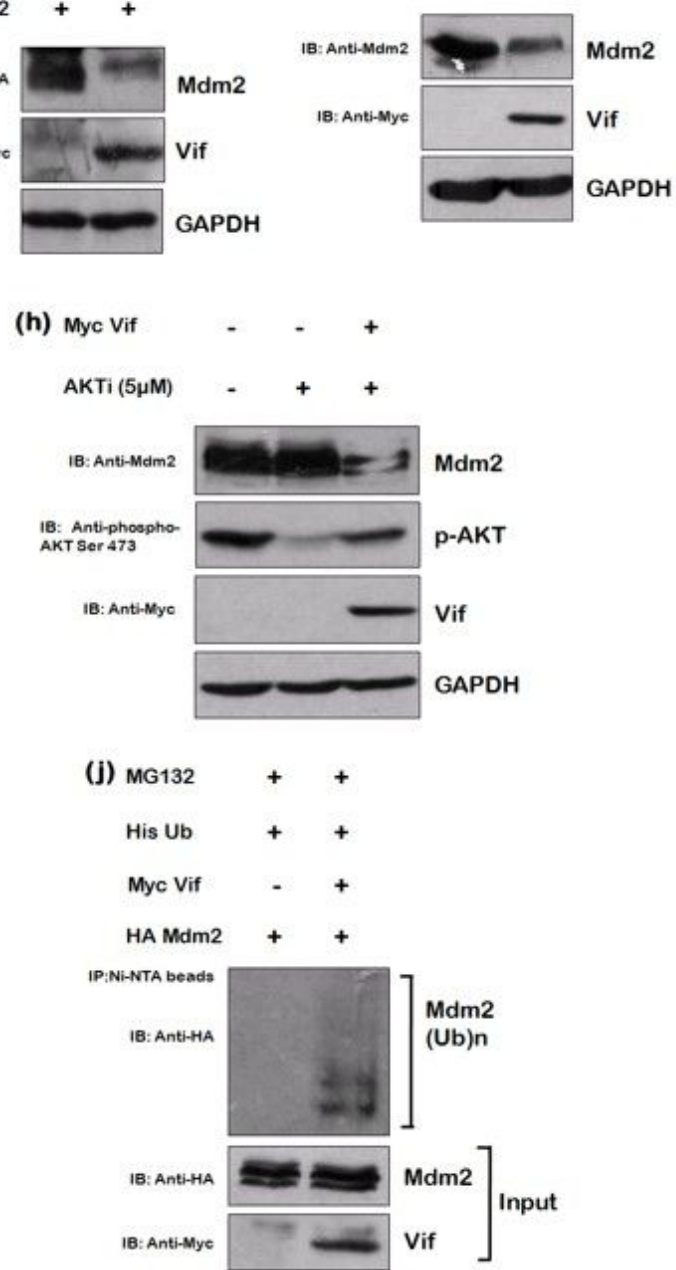
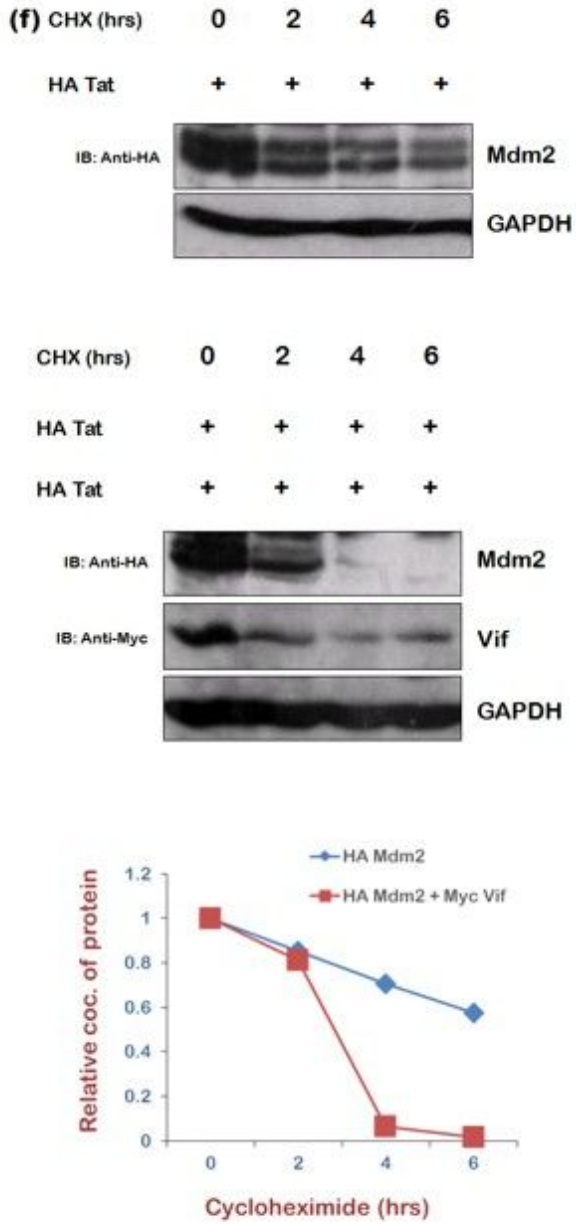

\section{Figure 1}

HIV-1 Vif induces phosphorylation of AKT at Ser473 while degrades Mdm2 via 26S proteasome. (a) HEK293T cells were co-transfected with HA AKT, HA Myr-AKT, HA KD-AKT and Myc Vif as shown for 24hrs.

Cell lysates were analyzed by western blotting with anti-HA, anti-phospho-AKT Ser473, anti-Myc and antiGAPDH antibodies. (b) HEK-293T cells were transfected with increasing amounts of Myc Vif expression plasmid for $24 \mathrm{hrs}$. Cell lysates were analyzed by western blotting with anti-AKT, anti-Myc, anti-phosphoAKT Ser473 and anti-GAPDH antibodies. (c) HEK-293T cells were transfected with Myc Vif expression plasmid and treated with AKTi $(5 \mu \mathrm{M})$ as indicated for $24 \mathrm{hrs}$. Cell lysates were analyzed by western blotting with anti-AKT, anti-Myc, anti-phospho-AKT Ser473 and anti-GAPDH antibodies. (d) HEK-293T cells were co-transfected with HA Mdm2 and Myc Vif expression plasmids for 24hrs. Cell lysates were 
subjected to western analysis using anti-HA, anti-Myc and anti-GAPDH antibodies. (e) HEK-293T cells were transfected with Myc Vif encoding plasmid for $24 \mathrm{hrs}$. Cell lysates were analyzed by western blotting with anti-Mdm2, anti-Myc and anti-GAPDH antibodies. (f) HEK-293T cells were transfected with HA Mdm2 and Myc Vif expression plasmids for 24hrs as indicated and treated with $\mathrm{CHX}(100 \mu \mathrm{g} / \mathrm{ml})$ for indicated time periods. Cells were lysed and cell lysates were subjected to SDS PAGE followed by western blotting using anti-HA, anti-Myc and anti-GAPDH antibodies. Densitometry analysis was performed using ImageJ and shown as line graph. (g) HEK-293T cells were co-transfected with HA Mdm2, HA Myr-AKT and Myc Vif encoding plasmids as shown for $24 \mathrm{hrs}$. Cells were lysed and cell lysates were subjected to SDS PAGE followed by western blotting with anti-HA, anti-Myc and anti-GAPDH antibodies. (h) HEK-293T cells were transfected with Myc Vif expression plasmid and treated with AKTi $(5 \mu \mathrm{M})$ for $24 \mathrm{hrs}$. Cell lysates were analyzed by western blotting with anti-Mdm2, anti-Myc, anti-phospho-AKT Ser473 and anti-GAPDH antibodies. (i) HEK-293T cells were co-transfected with HA Mdm2 and Myc Vif encoding plasmids for 24hrs. Cells were treated with MG132 $(20 \mu \mathrm{M})$ for 8hrs. Cell lysates were analyzed by western blotting with anti-HA, anti-Myc, and anti-GAPDH antibodies. GAPDH was used as loading control. (j) HEK-293T cells were co-transfected with His Ub, Myc Vif and HA Mdm2 for 24hrs. Cells were treated with MG132 (20 MM) for $8 \mathrm{hrs}$. Cell lysates were subjected to immunoprecipitation with Ni-NTA beads followed by western blotting with anti-HA antibody.

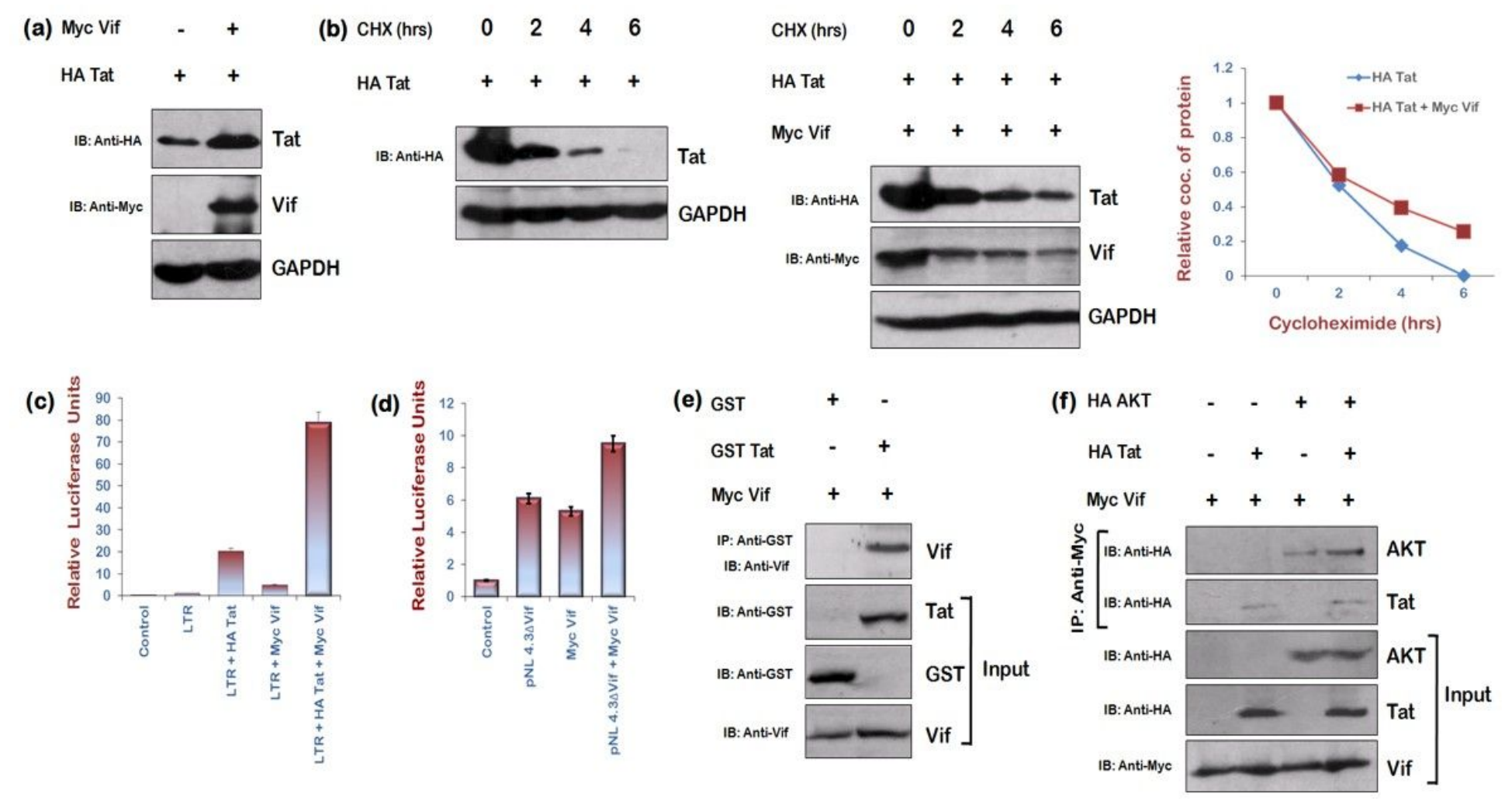

Figure 2

HIV-1 Vif increases the expression of Tat and interacts with it directly. (a) HEK-293T cells were cotransfected with HA Tat and Myc Vif plasmids as indicated for $24 \mathrm{hrs.} \mathrm{Cell} \mathrm{lysates} \mathrm{were} \mathrm{analyzed} \mathrm{by}$ western blotting using anti-HA, anti-Myc and anti-GAPDH antibodies. (b) HEK-293 T cells were transfected with HA Tat either alone or along with Myc Vif for 24 hrs. Cells were treated with Cycloheximide 
$(100 \mu \mathrm{g} / \mathrm{ml})$ for the indicated time periods. Cell lysates were subjected to western blot analysis using antiHA, anti-Myc and anti-GAPDH antibodies. GAPDH was used as loading control. Densitometric analysis was done by ImageJ and shown as line graph. (c) HEK-293T cells were co-transfected with HIV-1 LTR-luc reporter, Renilla luciferase, HA Tat and Myc Vif expression plasmids as indicated for $24 \mathrm{hrs}$. Cell were lysed and dual luciferase reporter assay was performed using luminometer. Relative luciferase activity was shown as bar graph. (d) Tzm-BI cells were transfected with Renilla luciferase, pNL4.3 $\Delta$ Vif and Myc Vif expression plasmids as indicated for $24 \mathrm{hrs}$. Cell were lysed and dual luciferase reporter assay was performed using luminometer. Relative luciferase activity was shown as bar graph. (e) GST Tat and GST alone bound with GST beads were incubated with in vitro synthesized Vif for $2 \mathrm{hrs}$ at $4^{\circ} \mathrm{C}$. Vif specific antiserum was used to probe the vif protein in western blot analysis. GST alone was used as negative control. (f) HEK-293T cells were transfected with HA Tat, HA AKT and Myc Vif encoding plasmids. After $24 \mathrm{hrs}$ of transfection, cell lysates were subjected to immunoprecipitation using anti-myc antibody bound agarose beads followed by western blotting using anti-HA antibody. 

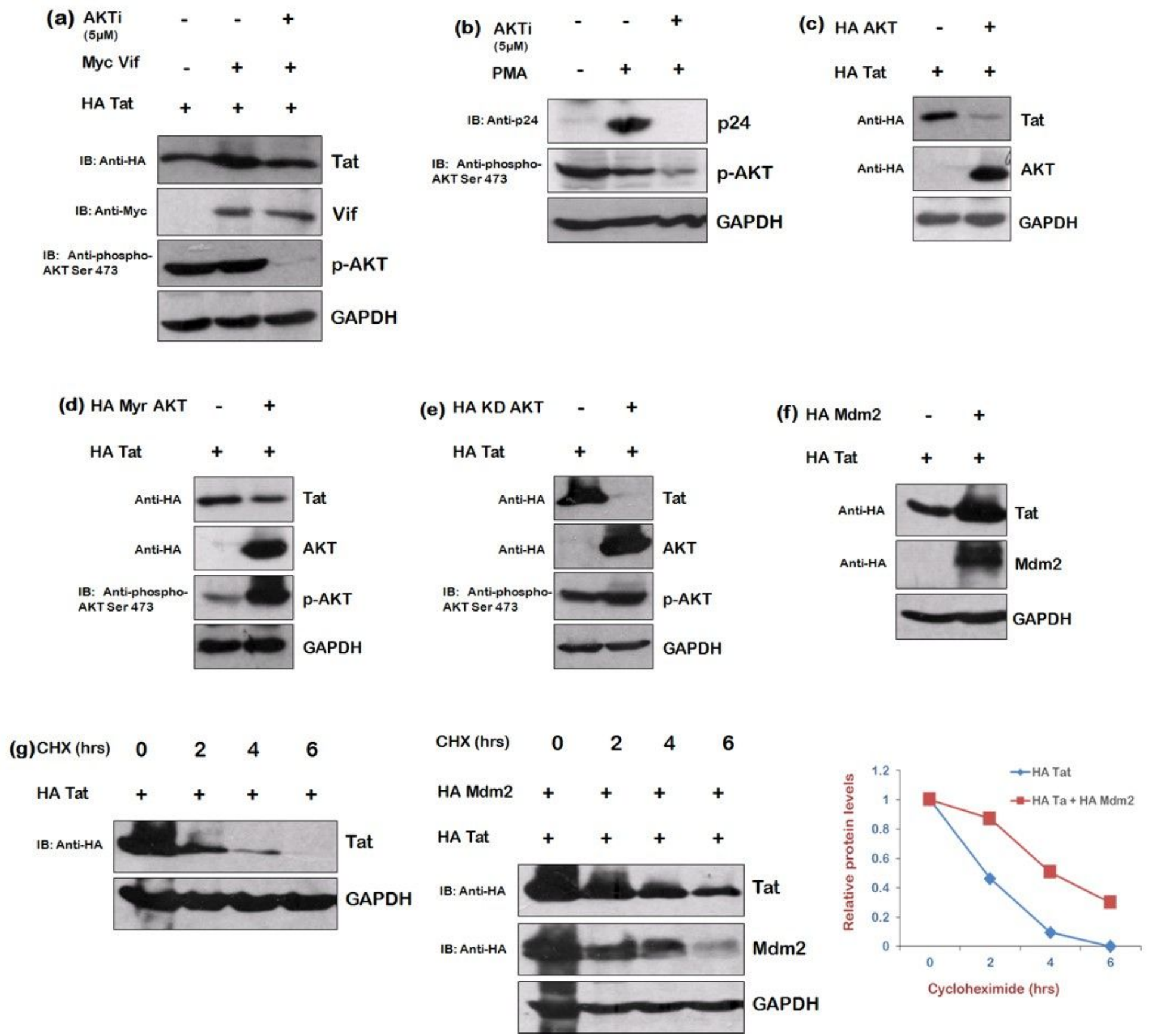

\section{Figure 3}

Mdm2 stabilizes the expression of HIV-1Tat. (a) HEK-293T cells were co-transfected with HA Tat and Myc Vif plasmids as indicated and treated with $\mathrm{AKTi}(5 \mu \mathrm{M})$ for $24 \mathrm{hrs}$. Cell lysates were analyzed by western blotting using anti-HA, anti-Myc, anti-phospho-AKT Ser473 and anti-GAPDH antibodies. (b) HIV-1 replication in U1 cells was induced by PMA and cells were treated with AKTi $(5 \mu \mathrm{M})$ for $24 \mathrm{hrs}$. Cell lysates were analyzed by western blotting using anti-p24, anti-phospho-AKT Ser473 and anti-GAPDH antibodies. (c) HEK-293T cells were transfected with HA Tat either alone or along with HA AKT for 24 hrs. Cell lysates were subjected to western blot analysis using anti-HA and anti-GAPDH antibodies. (d) HEK-293T cells were transfected with HA Tat either alone or along with HA Myr AKT for $24 \mathrm{hrs}$. Cell lysates were subjected to western blot analysis using anti-HA, anti-phospho-AKT Ser473 and anti-GAPDH antibodies. 
(e) HEK-293T cells were transfected with HA Tat either alone or along with HA KD AKT for $24 \mathrm{hrs}$. Cell lysates were subjected to western blot analysis using anti-HA, anti-phospho-AKT Ser473 and anti-GAPDH antibodies. (f) HEK-293T cells were transfected with HA Tat either alone or along with HA Mdm2 for 24 hrs. Cell lysates were subjected to western blot analysis using anti-HA and anti-GAPDH antibodies. (g) HEK-293 T cells were transfected with HA Tat either alone or along with HA Mdm2 for 24 hrs. Cells were treated with Cycloheximide $(100 \mu \mathrm{g} / \mathrm{ml})$ for the indicated time periods. Cell lysates were subjected to western blot analysis using anti-HA, anti-Myc and anti-GAPDH antibodies. GAPDH was used as loading control. Densitometric analysis was done by ImageJ and shown as line graph.

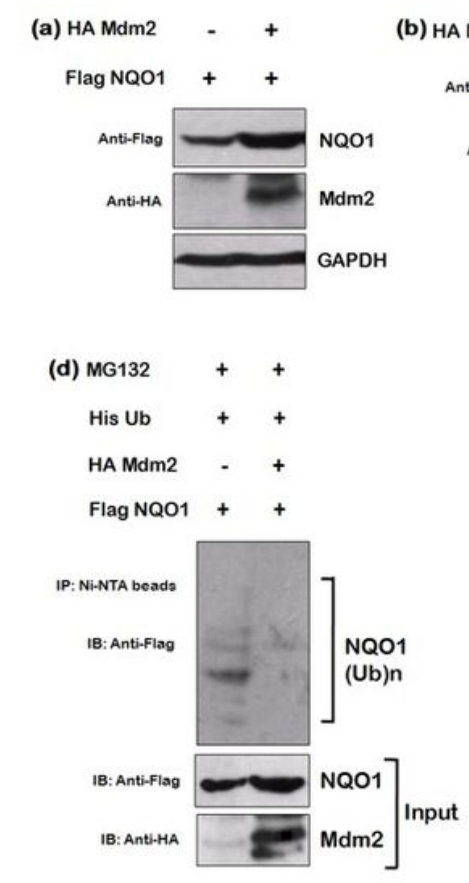

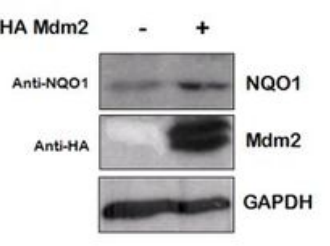

(c) $\mathrm{CHX}$ (hrs)

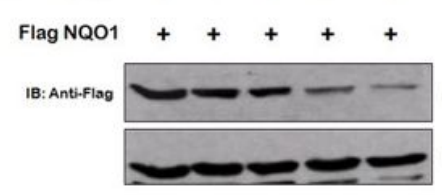

(e) HA Mdm2 - +

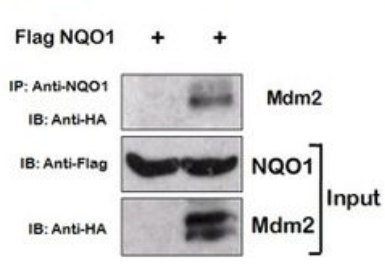

(g) Flag NQO1

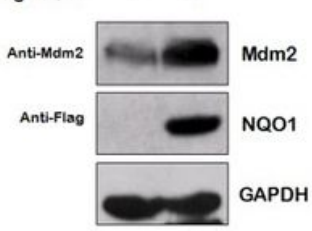

(f) Flag NQO1 - +

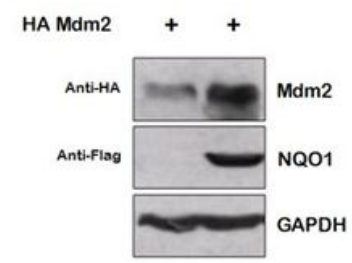

(h) Diminutol $(\mu \mathrm{M})$ Anti-Mdm2

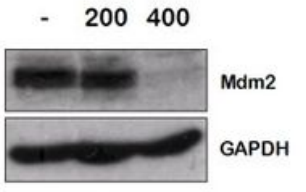

(j)

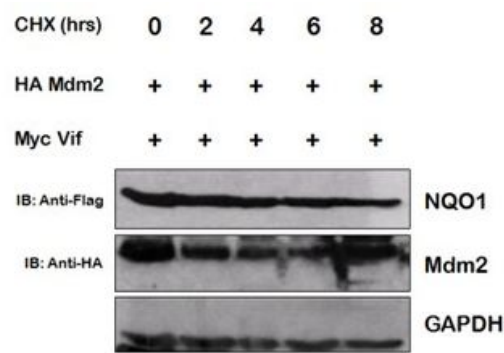

GAPDH
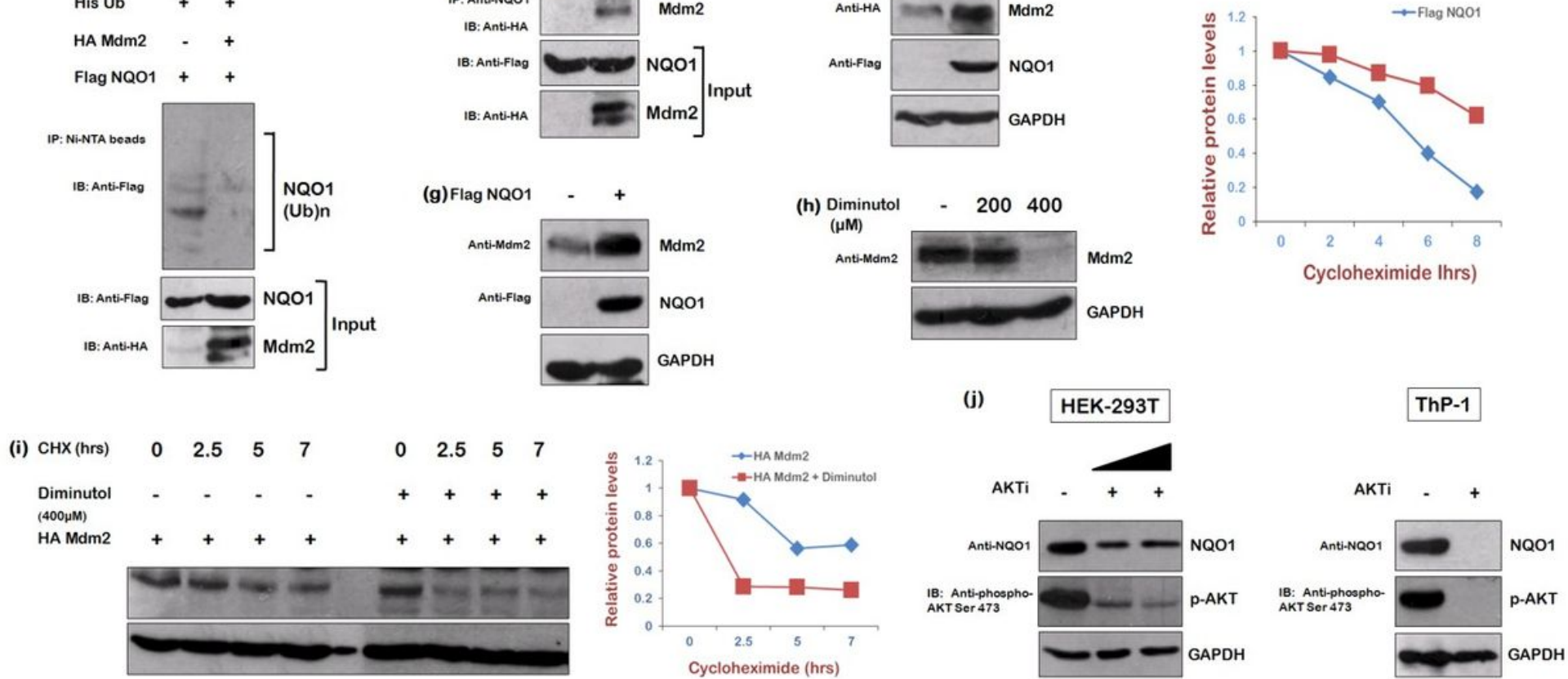

\section{Figure 4}

Mdm2 stabilizes the expression of NQ01 and vice-versa. (a) HEK-293T cells were co-transfected with Flag NQ01 and HA Mdm2 plasmids for 24 hrs. Cell lysates were analyzed by western blotting using antiHA, anti-Flag, and anti-GAPDH antibodies. (b) HEK-293T cells were co-transfected with HA Mdm2 plasmid for $24 \mathrm{hrs}$. Cell lysates were analyzed by western blotting using anti-NQ01, anti-HA, and anti-GAPDH antibodies. (c) HEK-293 T cells were transfected with Flag NQO1 either alone or along with HA Mdm2 for $24 \mathrm{hrs}$. Cells were treated with Cycloheximide $(100 \mu \mathrm{g} / \mathrm{ml})$ for the indicated time periods. Cell lysates were subjected to western blot analysis using anti-HA, anti-Flag and anti-GAPDH antibodies. Densitometric analysis was done by ImageJ and shown as line graph. (d) HEK-293T cells were co-transfected with His

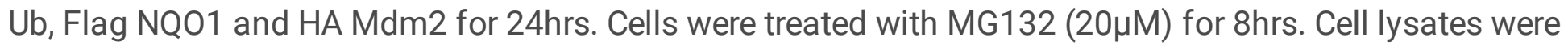


subjected to immunoprecipitation with Ni-NTA beads followed by western blotting with anti-Flag antibody. (e) HEK-293T cells were transfected with HA Mdm2 and Flag NQ01 encoding plasmids. After $24 \mathrm{hrs}$ of transfection, cell lysates were subjected to immunoprecipitation using anti-NQ01 antibody bound agarose beads followed by western blotting using anti-HA antibody. (f) HEK-293T cells were cotransfected with Flag NQ01 and HA Mdm2 plasmids for $24 \mathrm{hrs.} \mathrm{Cell} \mathrm{lysates} \mathrm{were} \mathrm{analyzed} \mathrm{by} \mathrm{western}$ blotting using anti-HA, anti-Flag, and anti-GAPDH antibodies. (g) HEK-293T cells were transfected with Flag NQ01 plasmid for 24 hrs. Cell lysates were analyzed by western blotting using anti-Mdm2, anti-Flag, and anti-GAPDH antibodies. (h) HEK-293T cells were treated with increasing doses of diminutol as indicated for $6 \mathrm{hrs}$ followed by western blotting using anti-Mdm2 and anti-GAPDH antibodies. (i) HEK-293 T cells were transfected with HA Mdm2 for $24 \mathrm{hrs}$ and treated with diminutol $(400 \mu \mathrm{M})$ for $6 \mathrm{hrs}$. Cells were treated with Cycloheximide $(100 \mu \mathrm{g} / \mathrm{ml})$ for the indicated time periods. Cell lysates were subjected to western blot analysis using anti-HA and anti-GAPDH antibodies. Densitometric analysis was done by ImageJ and shown as line graph. (j) HEK-293T and Thp-1 cells were treated with AKTi as indicated for 24 hrs. Cell lysated were subjected to western blotting using anti-NQ01, anti-phospho-AKT Ser473 and antiGAPDH antibodies. GAPDH was used as loading control.

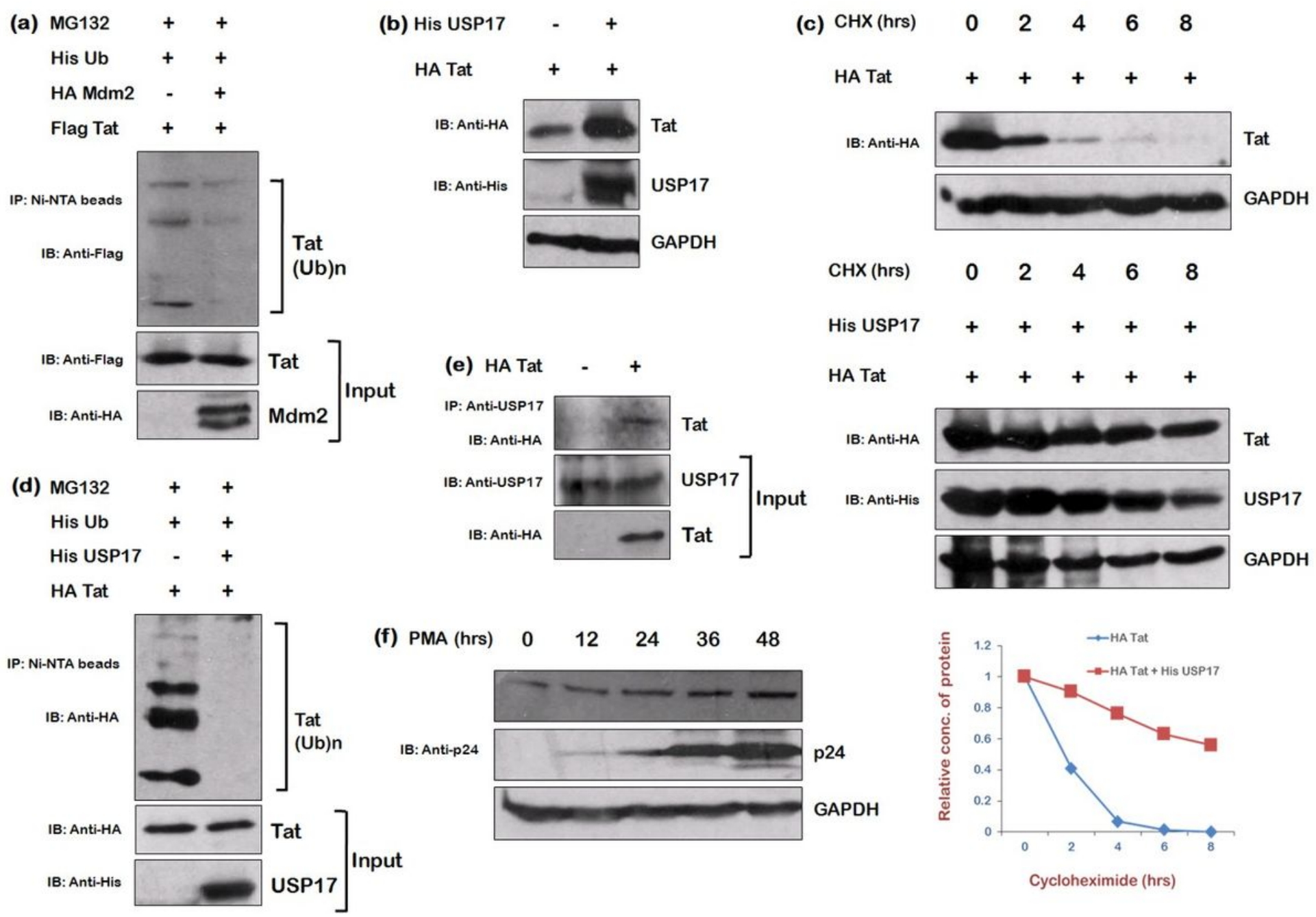

Figure 5 
USP17 stabilizes the expression of Tat. (a) HEK-293T cells were co-transfected with His Ub, Flag Tat and HA Mdm2 for 24hrs. Cells were treated with MG132 $(20 \mu \mathrm{M})$ for $8 \mathrm{hrs}$. Cell lysates were subjected to immunoprecipitation with Ni-NTA beads followed by western blotting with anti-Flag antibody. (b) HEK293T cells were co-transfected with HA Tat and His USP17 plasmids for $24 \mathrm{hrs}$. Cell lysates were analyzed by western blotting using anti-HA, anti-His, and anti-GAPDH antibodies. (c) HEK-293T cells were transfected with HA Tat either alone or along with His USP17 for 24 hrs. Cells were treated with Cycloheximide $(100 \mu \mathrm{g} / \mathrm{ml})$ for the indicated time periods. Cell lysates were subjected to western blot analysis using anti-HA, anti-His and anti-GAPDH antibodies. Densitometric analysis was done by ImageJ and shown as line graph. (d) HEK-293T cells were co-transfected with His Ub, HA Tat and His USP17 for 24hrs. Cells were treated with MG132 $(20 \mu \mathrm{M})$ for 8hrs. Cell lysates were subjected to immunoprecipitation with Ni-NTA beads followed by western blotting with anti-HA antibody. (e) HEK-293T cells were transfected with HA Tat encoding plasmid. After 24hrs of transfection, cell lysates were subjected to immunoprecipitation using anti-USP17 antibody bound agarose beads followed by western blotting using anti-HA antibody. (f) U1 cells were treated with PMA for indicated time periods to activate the latent HIV-1 replication. Cell lysates were analyzed by western blotting using anti-USP17, anti-p24, and anti-GAPDH antibodies. 
(a) HA Mdm2

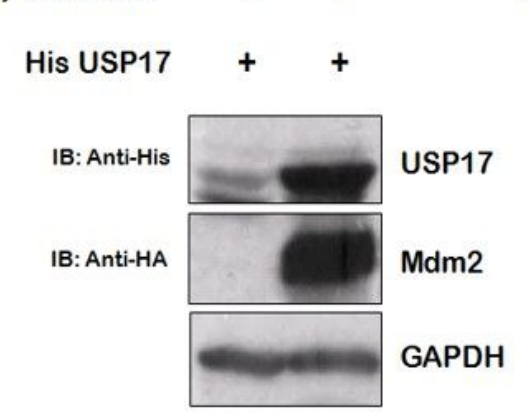

(d) MG132

His Ub

HA Mdm2

His USP17

IP: Ni-NTA beads

IB: Anti-USP17
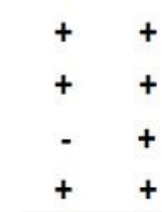

IB: Anti-His

IB: Anti-HA
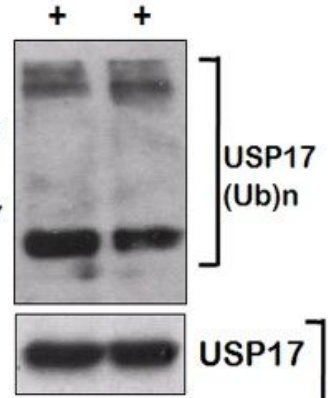

USP17

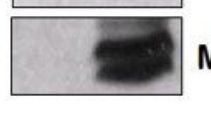

Mdm2

(f)

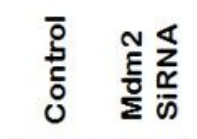

IB: Anti-USP17

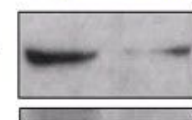

IB: Anti-Mdm2

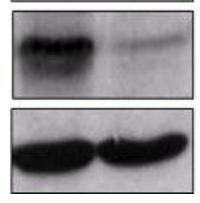

(b)

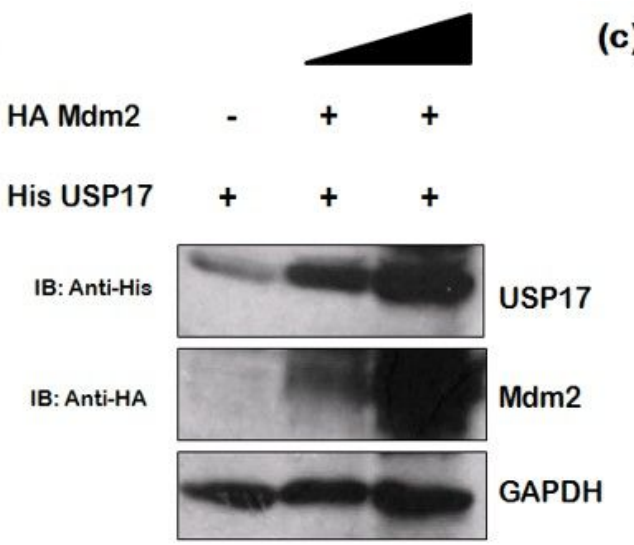

(e)

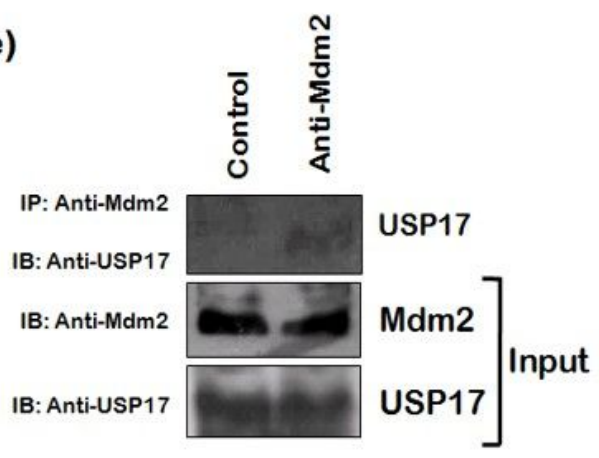

(g)
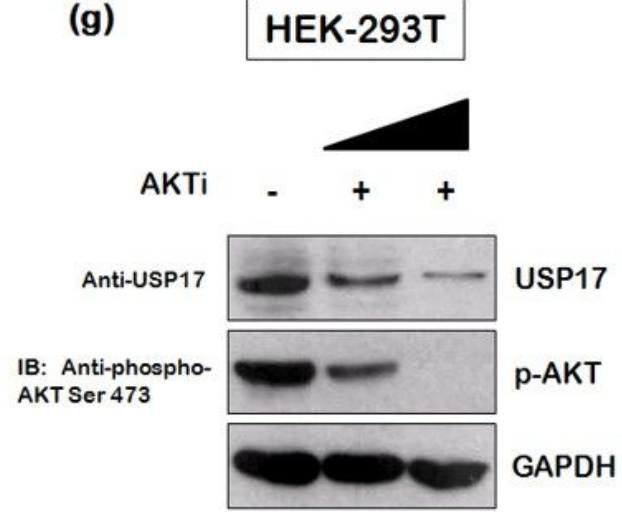

(c)

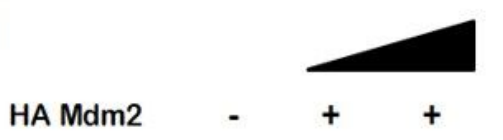

IB: Anti-USP17

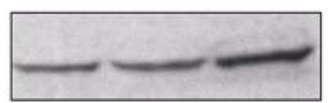

USP17

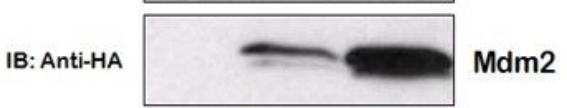

GAPDH

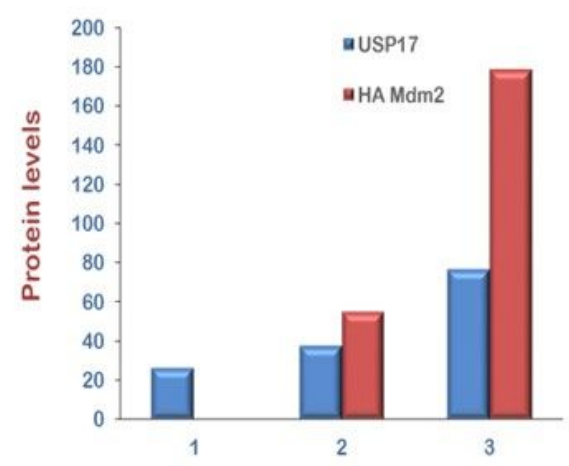

ThP-1

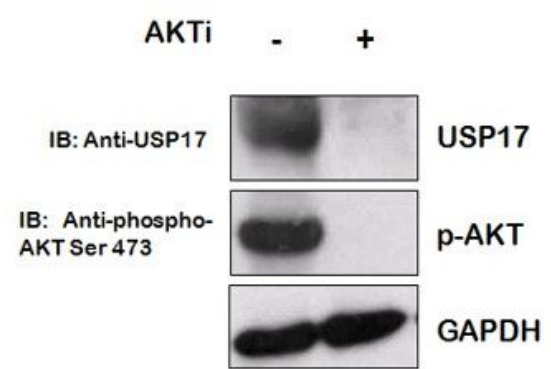

\section{Figure 6}

Mdm2 stabilizes the expression of USP17 (a) HEK-293T cells were co-transfected with His USP17 and HA Mdm2 plasmids for $24 \mathrm{hrs}$. Cell lysates were analyzed by western blotting using anti-His, anti-HA, and anti-GAPDH antibodies. (b) HEK-293T cells were transfected with His USP17 and increasing amounts of HA Mdm2 encoding plasmid. After $24 \mathrm{hrs}$ of transfection, cell lysates were subjected to western blotting using anti-His, anti-HA and anti-GAPDH antibodies (c) HEK-293T cells were transfected with increasing amounts of HA Mdm2 plasmid for $24 \mathrm{hrs}$. Cell lysates were analyzed by western blotting using anti-HA, anti-USP17, and anti-GAPDH antibodies. Densitometric analysis was done by ImageJ and shown as bar graph. (d) HEK-293T cells were co-transfected with His Ub, His USP17 and HA Mdm2 for 24hrs. Cells were treated with MG132 $(20 \mu \mathrm{M})$ for $8 \mathrm{hrs}$. Cell lysates were subjected to immunoprecipitation with Ni-NTA beads followed by western blotting with anti-USP17 antibody. (e) HEK-293T cell lysates were subjected to immunoprecipitation using anti-Mdm2 antibody bound agarose beads followed by western blotting using 
anti-USP17 antibody. (f) HEK-293 T cells were transfected with siRNA specific to Mdm2. After 48hrs of transfection, cell lysates were subjected to western blot analysis using anti-USP17, anti-Mdm2 and antiGAPDH antibodies. (g) HEK-293T and Thp-1 cells were treated with AKTi as indicated for $24 \mathrm{hrs}$. Cell lysates were subjected to western blotting using anti-USP17, anti-phospho-AKT Ser473 and anti-GAPDH antibodies. GAPDH was used as loading control.

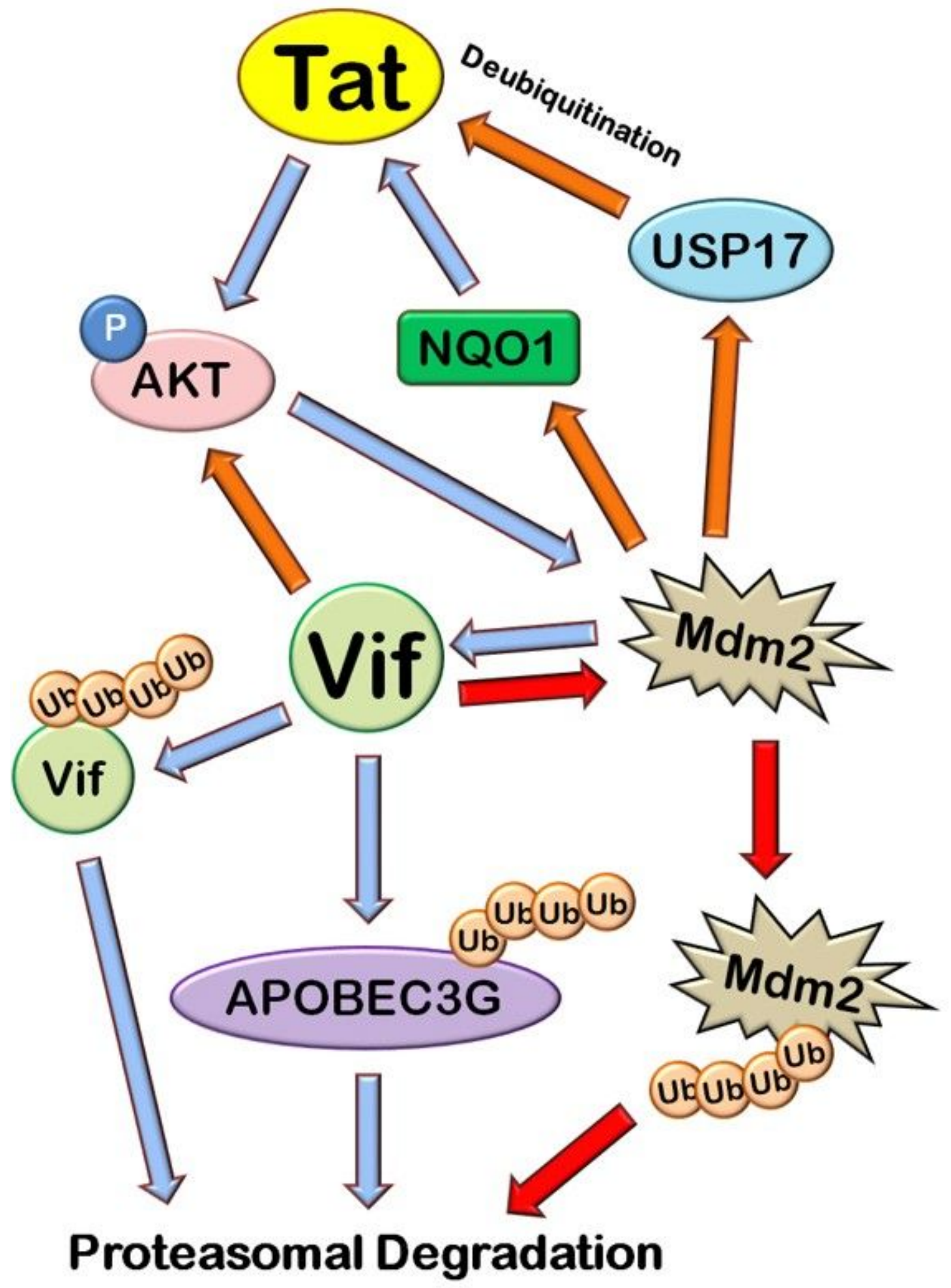

Figure 7 
Mechanistic model of HIV-1 Vif mediated up-regulation of Tat via AKT signaling pathway. When PI3/AKT signaling pathway gets activated, AKT gets phosphorylated and stimulates Mdm2 (downstream target of AKT). HIV-1 Tat induces AKT signaling pathway and increases the phosphorylation of AKT and Mdm2. Mdm2 stimulates ubiquitin-mediated proteasomal degradation of HIV-1Vif. Vif induces degradation of host anti-viral factor APOBEC3G by proteasomal machinery in ubiquitin-dependent manner. Vif also increases the phosphorylation of AKT similar to HIV-1 Tat but it induces proteasomal degradation of Mdm2 creating a negative feedback loop between Mdm2 and HIV-1 Vif. HIV-1 Vif increases the expression of Tat protein via AKT signaling pathway where Mdm2 acts as the mediator. Mdm2 increases the expression of Tat by inducing an increase in the levels of NQ01 which protects Tat from 20S proteasomal degradation and USP17 which stabilizes the levels of Tat by de-ubiquitination. (blue arrows previously known phenomena, golden yellow arrows - novel inductions, red arrows - novel inhibitions) 